\title{
Preliminary effects of pagoclone, a partial GABA agonist, on neuropsychological performance
}

\author{
Angela F Caveney' \\ Bruno Giordani' \\ George M Haig ${ }^{2}$ \\ 'Department of Psychiatry, University \\ of Michigan, Ann Arbor, MI, USA; \\ ${ }^{2}$ Neurosciences Development, Abbott \\ Laboratories, Abbott Park, IL, USA
}

Correspondence: Angela F Caveney Assistant Research Professor, Dept. of Psychiatry/Neuropsychology, University of Michigan, 2I0I Commonwealth, Suite C,Ann Arbor, MI 48105-5716, USA Tel +l 7349369273

Fax +I 7349369262

Email angif@umich.edu

\begin{abstract}
Pagoclone is a novel cyclopyrrolone that acts as a partial $\mathrm{GABA}_{\mathrm{A}}$ receptor agonist. Preclinical studies suggest that pagoclone may have clinical utility as an anxiolytic agent, as well as a reduced incidence of side-effects. The present study was conducted to determine whether pagoclone would affect healthy individuals' performances on neuropsychological measures as a function of dose within the projected therapeutic range. Twelve healthy adult subjects were randomly assigned to dosage groups in a 3-way crossover study. Participants were administered neuropsychological measures six hours following dosing on Day 1 and Day 6 of administration of the drug. Dose effects were noted on measures of alertness, learning, and memory and movement time. Significant effects were also noted on measures of alertness, learning and memory, information processing and psychomotor speed. Overall, the results of this small, preliminary study do not support a finding of behavioral toxicity for these doses of pagoclone. Rather, a pattern was found of transient and mild negative effects on learning and memory scores at the highest dose administered, though these changes were small and no longer evident by the sixth day of use.
\end{abstract}

Keywords: pagoclone, cyclopyrrolone, neuropsychological, memory, generalized anxiety disorder

\section{Introduction}

Behavioral toxicity refers to the extent to which a drug affects an individual's ability to perform the psychomotor and cognitive tasks of everyday life (Ramaekers 1998). At a significant level, behavioral toxicity may impair cognitive and psychomotor abilities to such an extent that the improvement of symptoms of psychological disorders is hindered. Generalized Anxiety Disorder (GAD) and Panic Disorder (PD) are among the most prevalent of psychiatric disorders. In the United States, as many as 5\% of people will experience GAD and 3.5\% will experience PD over the course of their lifetimes (American Psychiatric Association 2000). Agents currently used to treat GAD/PD include benzodiazepines, hypnotics, and anxiolytics. These medications are known to have variable, negative effects on memory, learning, motor function and cognition (Rickels and Rynn 2002). Thus, development of effective agents to treat GAD/PD with minimal negative cognitive effects is crucial.

Pagoclone (CI-1043, IP456) is a novel cyclopyrrolone that pharmacologically is a non selective partial $\mathrm{GABA}_{\mathrm{A}}$ receptor agonist (Lingford-Hughes et al 2005). A main feature of partial agonists is the limited degree to which they open the ion channel. In comparison, full agonists afford complete opening, allowing uninhibited influx of $\mathrm{Ca}^{+}$ions while occupying a relative low $(\sim 5 \%)$ percentage of receptors. Preclinical and clinical studies suggest that pagoclone may have clinical utility as an anxiolytic agent in the dose range of $0.15 \mathrm{mg}$ BID to $0.6 \mathrm{mg}$ BID, as well as reduced incidence of side-effects when compared to other conventional anxiolytics, specifically diazepam (Donevan et al 2000; Sandford et al 2001; Atack 2005). 
Pagoclone differs from benzodiazepines in that it does not emit a full electrophysiological response to GABA stimulation in vitro. Adverse events appear to be dose-related, with the most common side-effects reported as somnolence, dizziness, headache, asthenia, and lethargy, though cognitive effects have not yet been tested. In comparison, Zopiclone is also a partial GABA agonist cyclopyrrolone. Subtle and transient effects on memory and attention have previously been reported with zopiclone (Allain et al 1995; Stip et al 1999; Hemmeter et al 2000; Silva et al 2003).

The present study was conducted to determine whether pagoclone would affect a healthy individual's performance on neuropsychological measures of learning, memory, motor function and cognition, as a function of dose within the projected therapeutic range.

\section{Materials and methods Subjects}

A total of 12 healthy subjects (five men, seven women), aged 18-55 years gave their Internal Review Board (IRB) approved written consent after study procedures and possible side effects were explained to them. Participants were paid for their time. No significant differences for age were found across gender (Mean age men $=36.0, \mathrm{SD}=8.6$; Mean age women $=32.6, \mathrm{SD}=9.8$ ). Exclusion criteria were: 1 ) use of any central nervous system active or other potentially interfering medications during a 14-day period before the start of the study; 2) donation of a unit of blood within 30 days of the start of the study; 3) sexually active women not surgically sterile or using a reliable method of birth control; 4) history of significant adverse reaction to benzodiazepines or other anti-anxiety medications; 5) any clinically significant medical or psychiatric disorder; and 6) significant urine concentration of any drug that could interfere with the study.

\section{Neuropsychological measures}

\section{Sleepiness/alertness}

The Stanford Sleepiness Scale (Hoddes et al 1972) is a self-report, general measure of sleepiness consisting of a single rating based on seven statements with a higher score indicating increased sleepiness.

\section{Learning/memory}

Measures of learning and memory were derived from the Buschke Selective Reminding Test (BSRT) (Buschke and Fuld 1974). This test is a verbal word list learning task consisting of 6 trials of 24 words using selective reminding procedures. Subjects were asked to recall as many of the words from the word list as they could in any order. Each subsequent learning trial involved the selective presentation of only those items that were not recalled on the immediately preceding trial, but subjects were again asked to recall the whole list. The BSRT distinguishes between short-term and long-term components of memory by measuring recall of items that were not presented on a given trial. The following scores were measured from the BSRT: Immediate Recall (total number of words correctly remembered across all trails), Long Term Storage (LTS; total words recalled without the need for reminding), and Delayed Recall (total words recalled after a delay of approximately 20 minutes). Equivalent, alternate forms were available for this test.

\section{Psychomotor speed}

The Motor Screen subtest of the Cambridge Neuropsychological Test Automated Battery (CANTAB) (Morris 1987) represents a measure of visuomotor tracking requiring a subject to touch " $\mathrm{X}$ ' $\mathrm{s}$ " that randomly appear on a computer screen as quickly as possible. The primary score from this measure was the response time latency.

\section{Information processing efficiency}

Efficiency in information processing and sustained vigilance were measured with the Rapid Visual Information Processing task (RVP) from the computerized CANTAB battery. Subjects were given a two-minute training session to orient them to this task. In the four-minute test portion of this task, subjects were presented with three sequences of digits (eg, $3 * 5 * 7,2 * 4 * 6,4 * 6 * 8)$ and asked to press a button when the third digit of any of the target sequences appeared in a box on the screen. The target sequences remained on the screen to help subjects to remember them. Two primary scores were utilized from this measure: $\mathrm{A}^{\prime}$ and Mean Latency. $\mathrm{A}^{\prime}$ is a signal detection measure of sensitivity to the target regardless of the tendency to respond. Mean Latency reflects average response time for correct responses. Parallel forms were available for this test measure.

\section{Simple/complex attention}

Both simple (SRT; single target location) and choice (CRT, five target locations) reaction time tasks were administered to subjects with the computerized CANTAB system. In simple reaction time tests, subjects were asked to press a key as soon as they saw anything at all appear on the screen. In choice reaction time tests, subjects were asked to press a key only after they saw a specific visual stimulus appear on the screen. For each task, both Reaction Time (speed with which the subject releases a press pad in response to a stimulus) and 
Movement Time (time taken to touch the stimulus after the press pad has been released) were measured in milliseconds. Parallel forms were also available for this test.

\section{Psychomotor problem solving}

The Digit Symbol (Wechsler 1981) test is a code substitution task that measures processing speed and efficiency in psychomotor problem solving. The Digit Symbol test consists of 7 rows of small blank squares, each paired with a randomly assigned number from 1 to 9 . At the top of the page is a key that pairs each number with a different nonsense symbol. Subjects were required to fill in the blank spaces with the corresponding symbol as quickly as possible for 90 seconds. Parallel forms were also available for this test.

\section{Study design}

Subjects were entered into an open label (assessor blind), randomized, multiple-dose, 3-way crossover, pharmacokinetic dose-proportionality study. They received $0.15 \mathrm{mg}$ (low), $0.30 \mathrm{mg}$ (medium), or $0.60 \mathrm{mg}$ (high) doses of pagoclone every 12 hours for seven days with a seven-day washout between periods. Subjects were randomly assigned to one of three groups as they were screened for study inclusion. Groups differed by the order the drug doses were administered. Although capsule size varied, subjects were blinded to specific dosage. As subjects were randomly assigned, no effort was made to distribute gender evenly across groups, though two groups did each have two men and women and the third had three females and one male.

Three practice test sessions were administered prior to the baseline evaluations. Subjects were then tested at Baseline (the day prior to administration of the drug), Day 1, and Day 6 , in order to examine both acute and steady state effects. Neuropsychological testing was conducted approximately six hours after dosing on Days 1 and 6. Testing was completed at the same time of day for all evaluations (excluding practice). Six hours after dosing was close to the tmax for both pagoclone and the metabolite. Four trained examiners (two primary and two backup) completed the cognitive testing for the study. Examiners were blind with respect to treatment condition. Alternate test forms were pseudo-randomly assigned to each subject's testing sessions, such that no one subject saw the same form twice.

\section{Data analysis}

Change scores, calculated as Day 1 minus baseline and Day 6 minus baseline, were computed for all variables of interest. Data were analyzed using individual repeated measures
ANCOVA through SAS (PROC MIXED), using Least Squares Means. Analyses were done on 12 variables. Given the preliminary nature of this study, no attempt was made to adjust for the alpha level. Age was entered as a covariate and adjusted means from the ANCOVA are presented in this paper. The covariate, age, was found to be significant only for the three learning and memory measures (all $\mathrm{p}<0.05)$. Main effects were calculated for the variables of Gender (between effect), Dose (within effect). Day (within effect) and Order (between effect). Doses were coded as Low (0.15 mg), Medium (0.30 mg), or High (0.60 mg). Day was either Day 1 or Day 6. Order was designated as Order $1(0.15 \mathrm{mg}, 0.30 \mathrm{mg}$, $0.60 \mathrm{mg})$, Order $2(0.60 \mathrm{mg}, 0.15 \mathrm{mg}, 0.30 \mathrm{mg})$, and Order 3 (0.30 mg, $0.60 \mathrm{mg}, 0.15 \mathrm{mg}$ ).

Because this was primarily a preliminary pharmacokinetic study, it was decided that complete randomization was not necessary. Thus, three of six possible orders were used. Primary comparisons of interest were the overall changes in neuropsychological performance for Gender, Day, Order, and Dose, represented by main effects for each variable, and the interaction of Dose by Day to test for differential patterns of performance on each dose across the test days. Differences for least square means were examined for posthoc comparisons.

\section{Results}

Across all variables of interest, no significant findings were noted for the main effects of Order or Gender, or for the Dose by Day interaction. The results of analyses for the change scores for the main effects for both Day (Table 1) and Dose (Table 2) are reported in the sections below.

\section{Sleepiness/alertness}

On the Stanford Sleepiness scale, there were main effects for Day $(F=7.82, p=0.007)$ and for Dose $(F=4.20, p=0.021)$. Regardless of dose, subjects reported having a significantly greater increase in fatigue from baseline to Day 1 than from baseline to Day 6. The medium dose was associated with significantly higher self-report of fatigue than the lowest dose $(p<0.006)$. Average change scores for both the Dose and Day main effects, however, did not exceed one point.

\section{Learning/memory}

Three scores were analyzed from the Buschke Selective Reminding Test (ie, Immediate Recall, LTS, and Delayed Recall). A main effect for Day was noted on Delayed Recall $(F=811, p=0.006)$ and $\operatorname{LTS}(F=4.73, p=0.035)$. There was also a main effect for Dose for Immediate Recall 
Table I Change from baseline for all subjects: main effect of day

\begin{tabular}{|c|c|c|c|c|}
\hline \multirow[t]{2}{*}{ Test } & \multicolumn{4}{|l|}{ Day } \\
\hline & Day I & Day 6 & $\boldsymbol{F}$ & $\mathbf{p}<$ \\
\hline Stanford Sleepiness & 0.79 & -0.07 & 7.82 & 0.007 \\
\hline \multicolumn{5}{|l|}{ Buschke Selective Reminding } \\
\hline Immediate Recall & -2.80 & 0.34 & 2.68 & 0.108 \\
\hline Long Term Storage (LTS) & -7.63 & -0.21 & 4.73 & 0.035 \\
\hline Delayed Recall & -1.80 & -0.02 & 8.11 & 0.006 \\
\hline Motor Screen Latency & -37.90 & -46.17 & 0.07 & 0.798 \\
\hline \multicolumn{5}{|l|}{ RVP } \\
\hline$A^{\prime}$ & 0.00 & 0.02 & 8.27 & 0.006 \\
\hline Mean Latency & 2.21 & -38.84 & 12.22 & 0.001 \\
\hline \multicolumn{5}{|l|}{ Simple Reaction Time } \\
\hline Reaction Time & -13.07 & -17.07 & 0.84 & 0.364 \\
\hline Movement Time & -17.06 & -23.50 & 0.16 & 0.690 \\
\hline \multicolumn{5}{|l|}{ Choice Reaction Time } \\
\hline Reaction Time & -2.13 & -5.05 & 0.20 & 0.657 \\
\hline Movement Time & -5.93 & -7.26 & 0.00 & 0.959 \\
\hline Digit Symbol Test & 0.07 & 4.82 & 9.96 & 0.003 \\
\hline
\end{tabular}

Note: All scores have been adjusted with age as a covariate.

$(F=4.68, p=0.014)$, LTS $(F=5.34, p=0.008)$, and Delayed Recall $(F=3.71, p=0.032)$. An inspection of the BSRT variables on Table 1 reflects a consistent pattern of changes in performance essentially localized to Day 1, with Day 6 change scores essentially suggesting no change from baseline, hence a return to baseline functioning. Regarding Dose effects (Table 2), the decline from baseline in Immediate Recall was significantly greater for the high dose as compared to the low dose. For the LTS score, significantly greater declines from baseline were noted on both the medium and high doses as compared to the low dose.
For the Delayed Recall score, no change score differences were found between the Low Dose and the Medium Dose, though declines on the High Dose were significantly greater than the other two doses. Overall, there was a generally consistent pattern of no change to some improvement across change scores for the low dose, with declines in performance from baseline evident for the other two doses.

\section{Psychomotor speed}

Motor speed was measured with the Response Latency score from the Motor Screening task of the CANTAB battery.

Table 2 Change from baseline for all subjects: main effect of dose

\begin{tabular}{|c|c|c|c|c|c|}
\hline \multirow[t]{2}{*}{ Test } & \multicolumn{5}{|l|}{ Dose } \\
\hline & $0.15 \mathrm{mg}$ & $0.30 \mathrm{mg}$ & $0.60 \mathrm{mg}$ & $\boldsymbol{F}$ & $\mathbf{p}<$ \\
\hline Stanford Sleepiness & -0.14 & 0.94 & 0.27 & 4.20 & 0.021 \\
\hline \multicolumn{6}{|l|}{ Buschke Selective Reminding } \\
\hline Immediate Recall & 2.47 & -1.45 & -4.70 & 4.68 & 0.014 \\
\hline Long Term Storage (LTS) & 3.70 & -6.00 & -9.46 & 5.34 & 0.008 \\
\hline Delayed Recall & -0.18 & -0.43 & -2.10 & 3.71 & 0.032 \\
\hline Motor Screen Latency & -80.61 & -43.73 & -1.77 & 2.01 & 0.145 \\
\hline \multicolumn{6}{|l|}{ RVP } \\
\hline $\mathrm{A}^{\prime}$ & 0.01 & 0.00 & 0.01 & 1.55 & 0.223 \\
\hline Mean Latency & -11.98 & -23.07 & -19.90 & 0.31 & 0.731 \\
\hline \multicolumn{6}{|l|}{ Simple Reaction Time } \\
\hline Reaction Time & -17.56 & $-|8.8|$ & -8.85 & 2.06 & 0.138 \\
\hline Movement Time & -44.60 & -9.22 & -7.02 & 2.30 & 0.111 \\
\hline \multicolumn{6}{|l|}{ Choice Reaction Time } \\
\hline Reaction Time & -3.13 & -9.97 & 2.32 & 1.18 & 0.315 \\
\hline Movement Time & -53.22 & 26.86 & 6.57 & 3.46 & 0.039 \\
\hline Digit Symbol Test & 4.22 & 2.76 & 0.35 & 2.25 & 0.116 \\
\hline
\end{tabular}

Note: All scores have been adjusted with age as a covariate. 
Analyses of this variable demonstrated no significant main effects for Day or Dose.

\section{Information processing efficiency/ sustained attention}

For the A' score of the Rapid Visual Information Processing task, there was a main effect for Day $(F=8.27, p<0.006)$ and no effect for Dose. The increase in $\mathrm{A}^{\prime}$ was significantly higher on Day 6 as compared to Day $1(F=8.27, p=0.006)$, although the magnitude of this effect was small. Mean Latency analyses demonstrated a main effect of Day $(F=12.22, p=0.001)$ and no effect of Dose. Mean latencies increased slightly on Day 1, but declined on Day 6.

\section{Simple/complex attention}

For the Simple Reaction Time measures, there was no effect of Day or Dose on Reaction Time or Movement Time. For the Choice Reaction Time measures, there was no effect of DAY or DOSE on Reaction Time, but there was a main effect of DOSE on Movement Time $(F=3.46, p=0.39)$. Post-hoc comparisons of the effect of DOSE on Choice Reaction Time, Movement Time demonstrated that individuals in the Low Dose condition had faster Movement Time scores relative to baseline whereas subjects in the Medium Dose condition had slower Movement Time scores relative to baseline measures. The small increase in Movement Time for the High Dose condition was not significant $(p<0.06)$. A review of Table 2 reveals that the magnitude of the changes evident for the Dose effect (26-54 milliseconds) are relatively small in comparison to the standard deviation around the baseline mean (393.5 \pm 146.9 milliseconds).

\section{Psychomotor problem solving}

Digit Symbol analyses revealed a significant Day effect $(F=9.96, p=0.003)$ and no effect for Dose. Subjects demonstrated significantly improved test scores on Day 6 as compared to Day 1. An inspection of the data demonstrates that on Day 1 there was essentially no change from baseline performance. On Day 6, however, there was an increase in performance as compared to baseline, though this mean change did not exceed five points. This difference represents approximately 1.5 standard deviations from the baseline mean $(88.9 \pm 10.8)$.

\section{Discussion}

Overall, the results of this study suggest that subjects experienced very mild and transient negative effects on neuropsychological functioning when taking pagoclone, particularly at Day 1 and at doses above $0.15 \mathrm{mg}$. Although statistically significant, these findings were not generally clinically meaningful and most had dissipated by the second day (Day 6) of testing.

Specifically, with regard to dose of pagoclone, decrements in performance from baseline in learning and memory scores were generally greater for the high dose as compared with either the low or medium doses. For self-report of fatigue and the CRT movement time, the differences in the change scores from baseline were only noted for the medium as compared with the low dose. An inspection of the change scores generally suggested that speed and fatigue improved on the low dose, but declined on the medium dose, and to a lesser degree on the high dose. The clinical significance of these changes, however, must be considered. Across dose levels, the largest mean change did not exceed one point on the sleepiness scale, below what would be typically viewed as a clinically meaningful change on this measure. In addition, although statistically significant differences were evident in change scores on the Movement Time measure, an evaluation of mean scores and standard deviations for baseline suggest that the changes were not outside of expectation for basic test-retest variability.

These findings suggest that sedation effects with increased dose of pagoclone appear to be only very mild in nature, particularly since effects were not evident on more objective measures of motor speed and attention. Learning and memory scores, however, did suggest a fairly consistent pattern of somewhat lowered performance for the higher dose level, though additional analyses demonstrated that these changes were reasonably low in magnitude and no longer evident during testing on Day 6.

With respect to the day of testing, change scores from baseline reflected increased self-report of sleepiness and slower speed of information processing, along with poorer long term storage, on the first, as compared to the sixth, day of testing regardless of dose. Consistent with research demonstrating temporary memory impairments with the use of benzodiazepines (eg, Roth et al 1984; Lister 1985; Curran 1986; Ghoneim and Mewaldt 1990; Greenblatt et al 1991; Greenblatt 1992), these findings are suggestive of mild and short-lived sedation effects for pagoclone, regardless of Dose. In comparison to the decrements in select areas of neuropsychological performance noted on Day 1, improvements in performance were seen on Day 6 for psychomotor problem solving and target detection. These lowered performances on Day 1 followed by improvements on Day 6 on multiple tasks could reflect increased efficiency related to repeated 
task performance, though this is unlikely given three practices prior to baseline. The fact that lowered performance on several tasks on Day 1 did not significantly vary with drug dose raises the possibility of factors other than a direct effect of the pagoclone. It is possible, for example, that Day 1 effects may have been related to environmental or random factors other than pagoclone administration. However, the poorer performance on Day 1 was consistent across all three dosing periods suggesting a drug effect.

Unfortunately, the lack of a placebo control group, use of open label, small sample size and relatively large number of statistical analyses limit the overall conclusions that can be drawn from this preliminary study. The results do suggest, however, that across the six days of the study, behavioral toxicity of pagoclone was not a concern at these doses. Future research in larger groups of individuals and over longer periods of time is needed.

\section{Disclosure}

Study drug and project support were provided in part by a grant from Pfizer Pharmaceuticals Inc (Ann Arbor, MI, USA).

\section{References}

Allain H, Patat A, Lieury et al. 1995. Comparative study of the effects of zopiclone $(7.5 \mathrm{mg})$, zolpidem, flunitrazepam and a placebo on nocturnal cognitive performance in healthy subjects, in relation to pharmacokinetics. Eur Psychiatry, 10:S129-S135.

American Psychiatric Association. 2000. Diagnostic and Statistical Manual of Mental Disorders. IV-TR edn. American Psychiatric Association, Washington, DC.

Atack JR. 2005. The benzodiazepine binding site of GABA(A) receptors as a target for the development of novel anxiolytics. Expert Opin Investig Drugs, 14:601-18.

Buschke H, Fuld PA. 1974. Evaluating storage, retention, and retrieval in disordered memory and learning 5. Neurology, 24:1019-25.
Curran HV. 1986. Tranquillising memories: a review of the effects of benzodiazepines on human memory. Biol Psychol, 23:179-213.

Donevan S, Dougherty K, Haig G, et al. 2000. Investigator's Brochure of Pagoclone (CI-1043). Parke-Davis Pharmaceuticals, Ann Arbor, MI, USA, RR-X 720-04504.

Ghoneim MM, Mewaldt SP. 1990. Benzodiazepines and human memory: a review. Anesthesiology, 72:926-38.

Greenblatt DJ. 1992. Pharmacology of benzodiazepine hypnotics. J Clin Psychiatry, 53(Suppl):7-13.

Greenblatt DJ, Harmatz JS, Shapiro L, et al. 1991. Sensitivity to triazolam in the elderly. N Engl J Med, 324:1691-8.

Hemmeter U, Muller M, Bischof R, et al. 2000. Effect of zopiclone and temazepam on sleep EEG parameters, psychomotor and memory functions in healthy elderly volunteers. Psychopharmacology (Berl), 147:384-96.

Hoddes E, Zarcone V, Dement W. 1972. Development and use of Stanford Sleepiness Scale (SSS). Psychophysiology, 9:150.

Lingford-Hughes A, Wilson SJ, Feeney A, et al. 2005. A proof-of-concept study using $[11 \mathrm{C}]$ flumazenil PET to demonstrate that pagoclone is a partial agonist. Psychopharmacology (Berl), 180:789-91.

Lister RG. 1985. The amnesic action of benzodiazepines in man. Neurosci Biobehav Rev, 9:87-94.

Morris RG, Evenden JL, Sahakian BJ, Robbins T.W. (1987). Computer-aided assessment of dementia: comparative studies of neuropsychological deficits in Alzheimer-type dementia and Parkinson's disease. In: Stahl SM, Iversen SD, Goodman EC eds. Cognitive Neurochemistry. Oxford: Oxford University Press, pp 21-36.

Ramaekers JG. 1998. Behavioural toxicity of medicinal drugs. Practical consequences, incidence, management and avoidance. Drug Saf, 18:189-208.

Rickels K, Rynn M. 2002. Pharmacotherapy of generalized anxiety disorder. J Clin Psychiatry, 63:9-16.

Roth T, Roehrs T, Wittig R, et al. 1984. Benzodiazepines and memory. Br J Clin Pharmacol, 18(Suppl 1):45S-49S.

Sandford JJ, Forshall S, Bell C, et al. 2001. Crossover trial of pagoclone and placebo in patients with DSM-IV panic disorder. J Psychopharmacol, 15:205-8.

Silva A, Collao A, Orellana M, et al. 2003. Zopiclone, but not brotizolam, impairs memory storage during sleep. Neurosci Res, 47:241-3.

Stip E, Furlan M, Lussier I, et al. 1999. Double-blind, placebo-controlled study comparing effects of zopiclone and temazepam on cognitive functioning of insomniacs. Hum Psychopharmacol, 14:253-61.

Wechsler D. 1981. WAIS-R Manual. New York: Psychological Corporation. 